\title{
Clinical effects and safety of interventional therapy for parenchyma vascular malformation
}

\author{
M.J. Cai ${ }^{1}$, W.X. Liang ${ }^{2}$, W.W. Mai ${ }^{1}$, W.L. He ${ }^{1}$ and H.X. Kuang ${ }^{1}$ \\ ${ }^{1}$ Department of Radiology, \\ The Third Affiliated Hospital of Guangzhou Medical College, Guangzhou, \\ Guangdong, China \\ ${ }^{2}$ Department of Medical Ultrasound, \\ The Third Affiliated Hospital of Guangzhou Medical College, Guangzhou, \\ Guangdong, China \\ Corresponding author: M.J. Cai \\ E-mail: mingjincai@yeah.net
}

Genet. Mol. Res. 13 (1): 1735-1743 (2014)

Received September 18, 2013

Accepted October 15, 2013

Published January 22, 2014

DOI http://dx.doi.org/10.4238/2014.January.22.13

\begin{abstract}
Parenchyma vascular malformation (VM) is a common disease in modern society. Here, we investigated the clinical effects and safety of interventional therapy (IT) on the treatment of parenchyma VM. From January 1998 to December 2010, 31 patients with VM who elected IT were investigated, including 11 cases of venous VM and 20 cases of arteriovenous malformation. There were 19 males and 12 females, ranging from 12 to 51 years in age. VM often occurred in the four limbs and other areas, such as the trunk and reproductive organs. Under the guidance of digital subtraction angiography, vascular hardener was injected into the VM spot via percutaneous puncture. Then, embolotherapy was conducted via the transcatheter feeding artery. We found that, in all cases, the malformed vessels were completely or partially blocked. After treatment, the local swelling of vessels was alleviated and the diabrosis and bleeding ceased. The soft tissue lump shrank, then stiffened and became fixed.
\end{abstract}


There was no occurrence of severe intraoperative or postoperative complications in any patient. In summary, IT is an effective method for treating parenchyma VM, causes only a minor operative wound, and should be viewed as the first choice intervention.

Key words: Parenchyma; Vascular malformation; Interventional therapy

\section{INTRODUCTION}

Angioma and vascular malformation (VM) are common diseases in modern society, which most commonly occur in the skin and hypodermic tissues, and can then appear in the oral cavity mucous membrane and muscles. The disease rate is approximately $0.3-1 \%$ and the pathophysiological mechanism remains unclear. Many researchers have presumed that the disease is connected with the formation of blood vessels (Chang et al., 1999). According to the traditional classification of the characteristics of angioma endotheliocytes, Mulliken and Glowacki (1982) classified the angioma as a hemangioma and VM, whereas Jackson et al. (1993) classified VM as low flow rate VM (LFRVM) or high flow rate VM (HFRVM). Waner and Suen (1995) further classified VM into venous vascular malformation (VVM), arteriovenous malformation (AVM), and mixed type malformation, which are distinguished by the pathology of the vascular lesion and its hemodynamic characteristics. This classification allows much more meaningful clinical guidance for the choices of therapy methods and prognostic judgments. Therefore, it has become the formal classification method used by the International Vascular Diseases Association (IVDS). Currently, there are many methods for treating LFRVM, such as radiotherapy, laser therapy, surgery, and stiffening. Among these methods, the stiffening method has been identified as the first method of choice as it has the advantages of causing little scarring and infrequent complications (Lee and Chen, 2005; Zhi et al., 2008). The methods for treating HFRVM include operation, electrochemical therapy, and blood vessel inner embolism (Jackson et al., 1996; Yakes, 2004; Deng et al., 2010; Xue and Wang, 2011; Hatten and Bryant, 2012). In this study, 31 VM patients who were admitted to our hospital from January 1998 to December 2010 were treated with interventional therapy (IT) by embolotherapy with paracentesis or inner-artery stiffening. The clinical effect and safety of IT was investigated. We aimed to provide a basis for the further application of IT to the treatment of VM.

\section{MATERIAL AND METHODS}

\section{Patients}

We enrolled 31 patients at The Third Hospital Affiliated to Guangzhou Medical College (Guangzhou, China) from January 1998 to December 2010 in this study. There were 19 males and 12 females, ranging in age from 12 to 51 years (average $39 \pm 7.6$ years). The diseased regions were as follows: 17 cases affecting at least one of the four limbs, 8 cases affecting the trunk, 4 cases affecting a reproductive organ, and 2 cases affecting the neck. According to the previous classification method (Waner and Suen, 1995), 11 cases were VVM and 20 cases were AVM. All diagnoses were confirmed as VM based on disease history, clinical manifestations, ultrasound, magnetic resonance imaging (MRI), computed tomography (CT) three-dimensional (3D) angiography, and digital subtraction angiography (DSA). 


\section{Treatment}

Patients with different types of VM were treated using different methods. In this study, we treated VVM using embolotherapy with a percutaneous puncture hardener, while embolotherapy via an inner artery catheter was used for AVM. The embolization agents were 500-1400 $\mu \mathrm{m}$ polyvinyl alcohol (PVA), a gelatin sponge, and pinyangmycin lipiodol emulsion (PLE), in which $10 \mathrm{~mL}$ lipiodol emulsion was added to $8 \mathrm{mg}$ pinyangmycin that had been previously dissolved in 3-8 $\mathrm{mL}$ contrast media, and a syringe was then used to form an emulsion by repeated aspiration.

Stiffening embolotherapy via percutaneous puncture was performed as follows: under the guidance of DSA, a 20 G, 15-cm Chiba paracentetic needle (Cook Company; MO, USA) was inserted into the VM position. The vasography was used to initially determine the distribution and range of the thickening and circuitous abnormal vessel lump, and to assess the condition of the drainage vessels. Then, PLE was slowly injected into the abnormal vessel lump position for stiffening embolotherapy.

Stiffening embolotherapy via the percutaneous vascular inner-artery was conducted as follows: using Seldinger paracentesis intubation technology, a 4-5F Cobra or Yashiro catheter was chosen for the inner feeding artery vasography of the abnormal vessel lump. An assessment of the draining situation of the main feeding artery and vein toward the AVM was obtained. Based on the diseased region and range, highly selective catheterization was conducted to the targeted artery and a proper embolization agent was selected for embolotherapy. The diseased regions fed by multiple arteries were treated. In this study, 8 of 12 cases with AVM were injected with PLE and the other 4 cases were injected with a gelatin sponge pill and PVA that had been soaked with pinyangmycin. The selection of an embolization agent was based on the length of time over which the draining vein developed based on vasography for preventing ectopic embolism when embolization agents passed the orificium fistulae.

\section{Determination of curative effect}

Observations of the blood vessel lump, hardness, and corresponding symptoms were carried out six months after treatment. The total disappearance of the angioma and symptoms were classified as cured; the appearance of a significant diminution, partly callosity, no significant inflation when pressing the proximal part of the vein, or the disappearance of most symptoms were classified as effective; and no significant change or accretion of the blood vessel lump or related symptoms, or exacerbation of symptoms was classified as ineffective.

\section{RESULTS}

\section{DSA manifestation of abnormal blood vessels}

Direct paracentesis imaging of abnormal veins was facilitated by the retention of contrast media within vein-connected abnormal blood sinusoid tissues. The draining vein could be observed, whereas the feeding artery could not be imaged (Figures 1 and 2). The artery DSA imaging of the AVM showed obvious thickening and circuitous feeding images of the artery, whereas irregularly connected massive blood vessel lumps and the draining vein could be seen in the venous phase (Figure 3). 


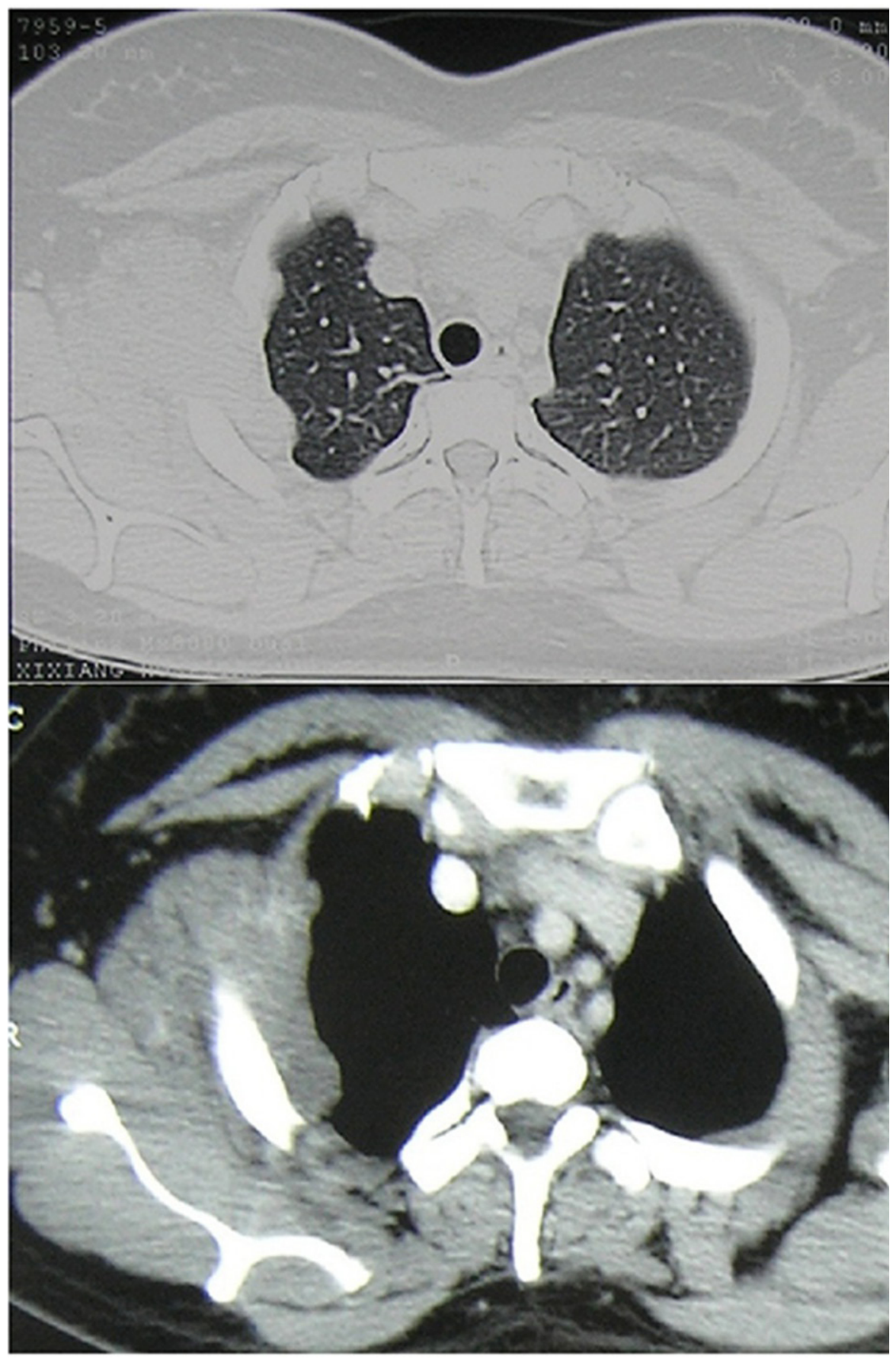

Figure 1. Soft tissue mass image could be seen at right-sided armpit, and increased when enhancing the intensity of scanning. According to this, venous malformation was diagnosed. 

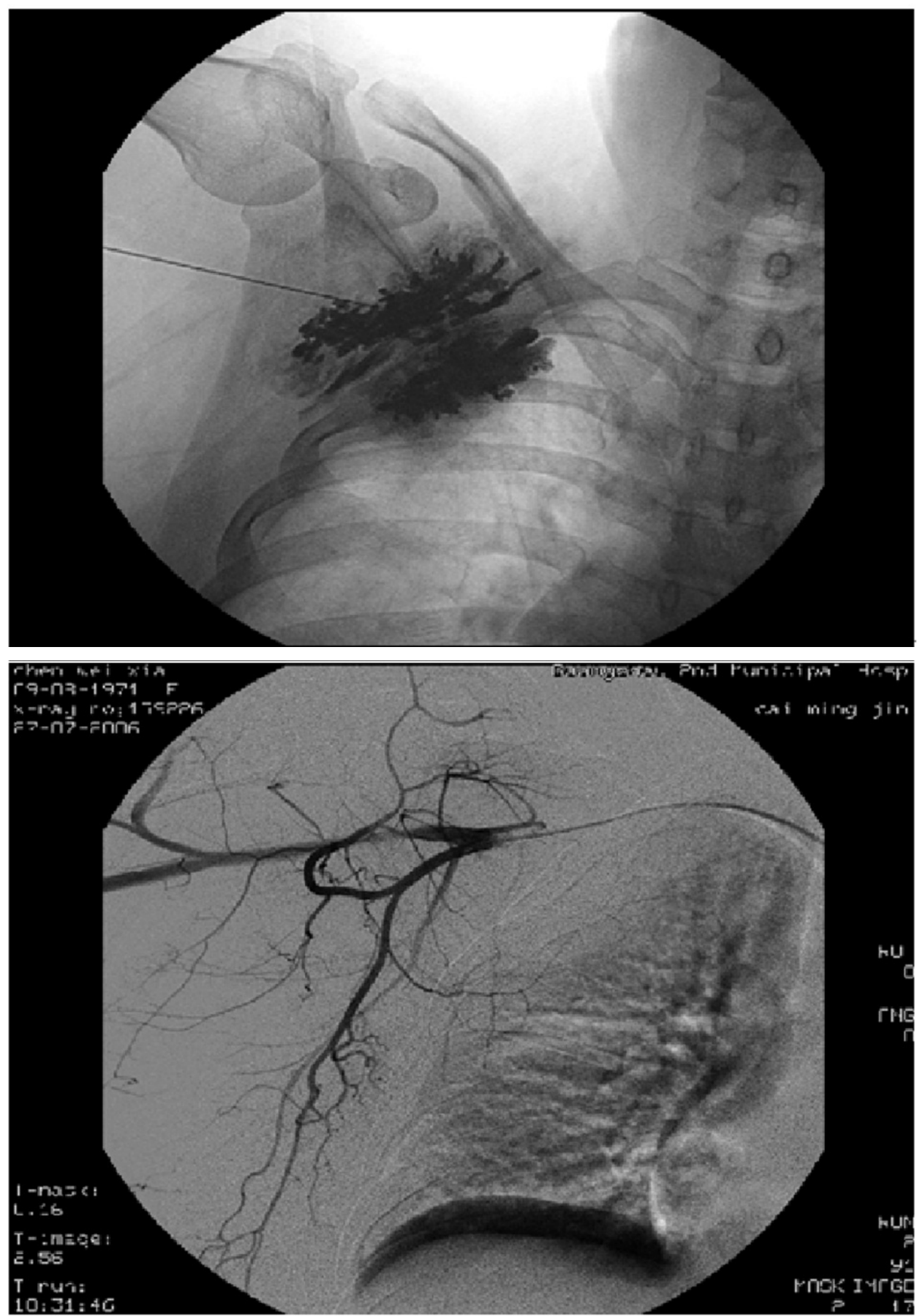

Figure 2. Direct puncture imaging was achieved by the contrast media entering and staying inside the abnormal blood sinusoid tissues. The draining veins could be seen in the figure, while there was no image of the feeding artery. 


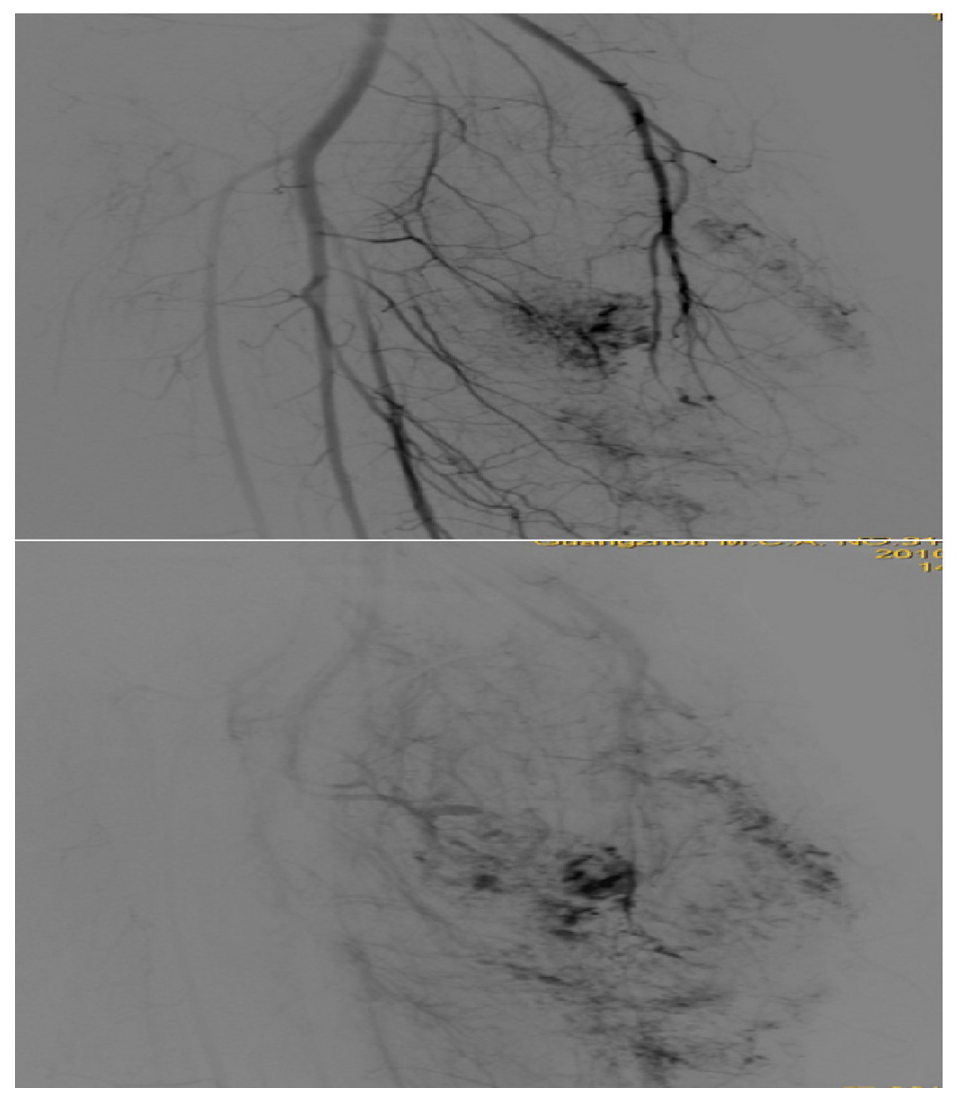

Figure 3. Thickening and circuitous feeding artery could be imaged in the arterial phase, while irregular interlocking massive blood vessel lump and draining veins could be seen in the venous phase.

\section{Postoperative reactions and symptoms}

All patients exhibited pain, swelling, fever, and pressing pain by 1-2 days after the operation, and these symptoms persisted for 3-7 days. Thereafter, the symptoms generally abated and eventually disappeared. Twelve patients, all undergoing stiffening embolotherapy of the percutaneous vascular inner artery, showed localized red swelling and brightness of the skin, but no severe symptoms, such as cutaneous necrosis, occurred.

\section{Clinical effects}

The 3-12-month follow-up interviews (all performed by phone call or personal checkup) indicated that 31 cases were cured without ineffective results, among which 16 patients accepted only one treatment and the other 15 patients accepted treatment 2-4 times. The pain symptoms subsided or disappeared, the soft tissue mass decreased, and the pathological blood vessels significantly decreased (Figure 4). 


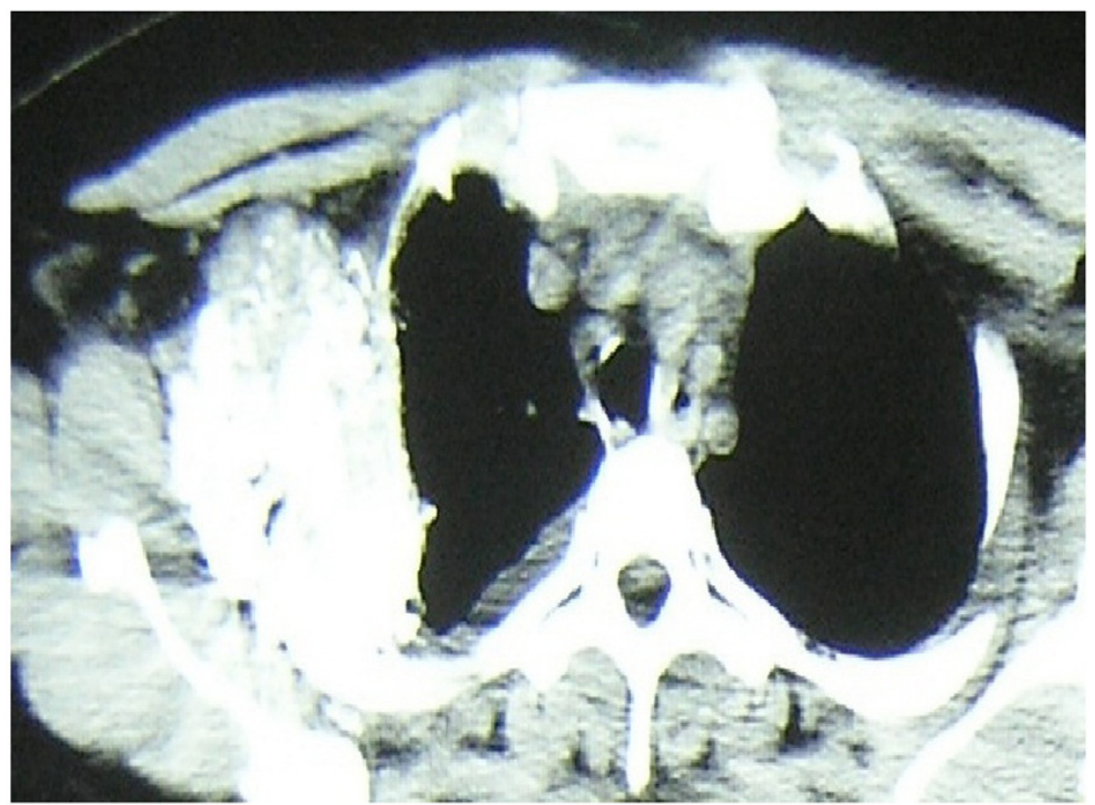

Figure 4. CT recheck after 1 month from treatment: soft tissue mass decreased and filled with lipiodol; pathological blood vessel was observed.

\section{DISCUSSION}

VM is caused by geogenous abnormal vessel formation, and the disease position can increase proportionally with age and does not spontaneously decrease. According to the tissue structure, VM can be classified into capillary VM, venular malformation, VVM, or AVM (Wane and Suen, 1995). The clinical appearance of VM can change according to the position, size, and range of the diseased position. VM appears on the surface of the body as easily ruptured tissue, tissue that has undergone repeated bleeding, or a secondary infection, whereas VM in muscles shows characteristics such as a local soft tissue mass, limb thickening or deformation, skeleton destruction, or pathological fractures. AVM can be indicated by blood vessel beating, as it leads to vascular murmur.

VM cases should be treated differently according to their classification. Since AVM is mainly caused by the direct connection of the ecstatic artery and vein, stiffening embolotherapy of the percutaneous vascular inner artery is the preferred therapeutic method. In artery imaging toward the diseased AVM position, a significant return to the feeding artery and vein would be seen. This is especially apparent in AVM of the limbs, which normally have multiple feeding arteries and an abnormal blood vessel lump in the venous phase. According to the diseased position and blood-feeding situation, highly selective catheterization was conducted to the feeding artery by injecting an embolization agent to cure the pathological vessels. This operation could block the flow of blood that feeds the tumor, which would gradually block and decrease the abnormal blood vessel lump (feeding artery and draining vein) and effectively control, or sometimes cure, the development of disease. Currently, there are many materials for inner artery embolotherapy of VM (Iwanaka et al., 1993; Kuniaki, 1993; Ogita et al., 
1993; Yakes et al., 1996, 1997), and, in this study, PLE and 500-1400 $\mu \mathrm{m}$ PVA were chosen. Pinyangmycin has the biological effects of promoting the hardening of blood vessels and has anti-tumor-like effects on hyperplastic vascular endothelial cells. Lipiodol was used as a vehicle to help the drug stay in the diseased position longer. After the injection of PLE, cell hyperplasia was inhibited immediately and the blood vessel lump would then shrink because of the lack of blood feeding for growth (Kong et al., 2003; Zeng et al., 2004; Chen et al., 2008; Guan et al., 2011). If arteriovenous fistulae occurs in AVM, 500-1400 $\mu \mathrm{m}$ PVA could be chosen for the embolism of orificium fistulae, and then PLE could be injected. Repeated low-dose embolotherapy could be accepted based on safety considerations for patients with a wide VM range and abundant blood vessels. For patients without good results of vascular inner artery embolotherapy, direct local injection of hardener could be incorporated into the therapy. In this study, 8 patients received 1-3 rounds of local hardener therapy treatment, and all achieved good results.

Since VM is composed of several expanding veins with different sizes, stiffening embolotherapy via percutaneous puncture is the main therapy for VM. All 11 patients with VVM were given paracentesis tumor cavity contrast before the treatment, and the drug was injected under the perspective condition. This approach made the size, range, and draining situation of the blood vessel lump much clearer, so that the dosage, blood concentration range, and the judgment of therapeutic effects could be determined more comprehensively with greater safety and efficacy. Before performing stiffening embolotherapy, the position, size, and relationship with the surrounding tissues of the tumor should be evaluated by CT or MRI so that the puncture needle insertion location and the range of dosage can be optimized, which would make the perfusion of the hardener more effective. The important point is that the injection of hardener into the abnormal vein lumen should be guaranteed.

In this study, all patients showed localized red swelling and skin pains in the first stage. After about 1 week, these symptoms receded automatically without special treatments. In order to decrease side effects, amcinonide could be used for the abatement of aseptic inflammation. Although no severe complications occurred in our study, complications are unavoidable in some cases. Therefore, prevention steps were designed and informed consent was obtained from the patients or their relatives. The key for prevention was to ensure that the needle pinhead was set inside the angioma intracavity or that the catheter was ultraselectionally inserted into the feeding artery. If necessary, a microtubule could be used and a small amount of contrast agent can be injected for confirmation. The injection speed should be slow and the dosage should be optimized to prevent misembolism caused by injecting too much hardener into the draining vein or normal tissues. If visualization reveals the existence of many draining veins, or relatively large arteriovenous orificium fistulae, the treatment should not be attempted.

\section{REFERENCES}

Chang J, Most D, Bresnick S, Mehrara B, et al. (1999). Proliferative hemangiomas: analysis of cytokine gene expression and angiogenesis. Plast. Reconstr. Surg. 103: 1-9.

Chen Y, Li Y, Zhu Q, Zeng Q, et al. (2008). Fluoroscopic intralesional injection with pingyangmycin lipiodol emulsion for the treatment of orbital venous malformations. AJR Am. J. Roentgenol. 190: 966-971.

Deng W, Huang D, Chen S, Zhang X, et al. (2010). Management of high-flow arteriovenous malformation in the maxillofacial region. J. Craniofac. Surg. 21: 916-919.

Guan JY, He XF, Chen Y, Zeng QL, et al. (2011). Percutaneous intratumoral injection with pingyangmycin lipiodol emulsion for the treatment of recurrent sacrococcygeal chordomas. J. Vasc. Interv. Radiol. 22: 1216-1220. 
Hatten BW and Bryant E (2012). Bleeding scrotal arteriovenous malformation. J. Emerg. Med. 42: e133-e135.

Iwanaka T, Tsuchida Y and Kawarasaki H (1993). Corticocteroid treatment for infantile hemangiomas of the eyelid and orbit . Jpn. J. Pediatr. Surg. 25: 427.

Jackson IT, Carreno R, Potparic Z and Hussain K (1993). Hemangiomas, vascular malformations, and lymphovenous malformations: classification and methods of treatment. Plast. Reconstr. Surg. 91: 1216-1230.

Jackson JE, Mansfield AO and Allison DJ (1996). Treatment of high-flow vascular malformations by venous embolization aided by flow occlusion techniques. Cardiovasc. Intervent. Radiol. 19: 323-328.

Kong WD, Yi YH, He XF, Chen Y, et al. (2003). Intravascular administration of pingyangmycin lipiodol emulsion for Klippel-Trenaunay syndrome: clinical analysis of 7 cases. Di Yi Jun. Yi Da Xue Xue Bao 23: 1109-1111.

Kuniaki O (1993). Trearment of infantile hemangioma from the dermatological point of view. Jpn. J. Pediatr. Surg. 25: 439.

Lee $\mathrm{CH}$ and Chen SG (2005). Direct percutaneous ethanol instillation for treatment of venous malformation in the face and neck. Br. J. Plast. Surg. 58: 1073-1078.

Mulliken JB and Glowacki J (1982). Hemangiomas and vascular malformations in infants and children: a classification based on endothelial characteristics. Plast. Reconstr. Surg. 69: 412-422.

Ogita S, Tsuto T, Nakamura K, Deguchi E, et al. (1993). OK-432 thrapy for cystic hygroma and cavernous lymphangioma. Jpn. J. Pediatr. Surg. 25: 371-376.

Waner M and Suen JY (1995). Hemangiomas and vascular malformtions of the head and neck. Oncology 9: 989-997.

Xue L and Wang XK (2011). Application of electrochemical therapy in high-flow vascular malformation in maxillofacial region. Zhonghua Zheng. Xing. Wai Ke. Za Zhi 27: 28-30.

Yakes WF (2004). Endovascular management of high-flow arteriovenous malformations. Semin. Intervent. Radiol. 21: 49-58.

Yakes WF, Rossi P and Odink H (1996). How I do it. Arteriovenous malformation management. Cardiovasc. Intervent. Radiol. 19: 65-71.

Yakes WF, Krauth L, Ecklund J, Swengle R, et al. (1997). Ethanol endovascular management of brain arteriovenous malformations: initial results. Neurosurgery 40: 1145-1152.

Zeng Q, Li Y, Chen Y, Ouyang Y, et al. (2004). Gigantic cavernous hemangioma of the liver treated by intra-arterial embolization with pingyangmycin-lipiodol emulsion: a multi-center study. Cardiovasc. Intervent. Radiol. 27: 481485.

Zhi K, Wen Y, Li L and Ren W (2008). The role of intralesional Pingyangmycin in the treatment of venous malformation of facial and maxillary region. Int. J. Pediatr. Otorhinolaryngol. 72: 593-597. 